\title{
Die Grippe-Epidemie 1918/1919 in der schweizerischen Armee
}

\author{
Von Walter Nussbaum
}

\section{Einleitung}

Wenn ich mich mit der Grippe-Epidemie in der schweizerischen Armee der Jahre 1918 und 1919 befaßte, so lag die Veranlassung dazu darin, daß ich als Sanitätsoffizier die Leistungsfähigkeit unseres Sanitätsdienstes im einzigen erlebten und wirklich durchgespielten Ernstfall dieses Jahrhunderts untersuchen wollte. Weil gleichzeitig die Zivilbevölkerung befallen war, konnte man auch die Schwierigkeiten sehen, die ein moderner Krieg mit sich brächte, wo mit Sicherheit sowohl die Armee wie auch die Zivilbevölkerung betroffen würde.

\section{Frühere Grippe-Epidemien}

Die Grippe ist eine Infektionskrankheit, die seit dem Altertum bekannt ist und die in ihrer schweren Form ungefähr alle Menschenalter einmal die zivilisierte Welt befällt und im Mittelalter und in der Neuzeit noch neben den Pest- und Cholera-Epidemien auftrat. Der für diese Krankheit gebräuchliche Ausdruck Influenza wurde 1743 in England geprägt. Die letzte große Influenza-Epidemie befiel die Schweiz in den Jahren 1889-1895. Sie wurde vom damaligen Direktor des eidgenössischen Gesundheitsamtes, Dr. Friedrich Schmid, sehr gut studiert und anhand von Mitteilungen von über 700 Ärzten für die ganze Schweiz zusammengestellt. Die Bakteriologie steckte damals noch in den Kinderschuhen, waren doch erst in den 80er Jahren die wichtigsten bakteriologischen Entdeckungen, wie z. B. die des Kochschen Tuberkelbazillus, gemacht worden. Immerhin kam man allgemein damals zur Auffassung, daß es sich bei der Influenza um eine Infektionskrankheit handle, obschon bei gewissen Ärzten noch altertümliche Vorstellungen herrschten, wie daß diese Krankheit durch sogenannte Miasmen, d.h. von krankmachenden Dünsten, erzeugt würde. Im Jahre 1892 entdeckte dann der Bakteriologe Pfeiffer bei Grippefällen den nach ihm benannten Influenza-Bazillus, der noch bis 1940 erwähnt wurde, jedoch nicht der Erreger der Grippe ist. Eine eigentliche Prophylaxe konnte in den 90er Jahren so 
wenig wie 1918 durchgeführt werden, und das einzig Mögliche war wohl die Vorschrift des waadtländischen Kantonsarztes, der nach Möglichkeit ein Versammlungsverbot anordnete, um die Ansteckung zu verhindern. Dieselben Maßnahmen wurden dann auch 1918/19 wieder angeordnet.

Was das Ausmaß der Epidemie der 90er Jahre anbelangt, war diese sehr viel kleiner als dann im Jahre 1918. Im ersten Jahr der Epidemie gab es vom Dezember 1889 bis in den Winter 1890 in der Schweiz 2669 Grippe-Tote, was bei einer Bevölkerungszahl von etwa 2,9 Millionen einer Mortalität von $0,89 \%$ entspricht. Wenn wir diese Epidemie mit derjenigen von 1918/19 vergleichen, so müssen wir feststellen, daß innerhalb von 12 Monaten in der Schweiz gegen 24000 Leute an der Grippe gestorben sind, was einer Mortalität von über 6\% entspricht. Vom Standpunkt des Epidemiologen aus gesehen ist die Grippe eine der interessantesten Infektionskrankheiten und mit keiner andern zu vergleichen. Es herrscht eine unglaubliche Variabilität der Virulenz des Erregers, die sich in der Epidemie der 90er Jahre ebenso zeigte wie dann in derjenigen von 1918 und 1919. Beiden Epidemien war die starke territoriale Verschiedenheit der Grippe-Virulenz gemein. Schmid rechnete die Mortalität jedes Amtsbezirkes auf 10000 Einwohner der Schweiz um und zeichnete sie in eine Karte ein. Dabei fällt uns z. B. für das erste Jahr der Amtsbezirk Saanen im Berner Oberland auf, der, umgerechnet auf 10000 Einwohner, eine Todesfallquote von 47,0 aufwies, während das benachbarte Gebiet des waadtländischen Amtsbezirks Pays-d'Enhaut mit Château-d'CEx eine Todesfallquote von nur 0 hatte, obschon die beiden Gebiete im gleichen Tal liegen, die Bevölkerung gleiche soziologische Zusammensetzung hat und auch der Verkehr miteinander sehr rege ist. Solche Unterschiede, nicht ganz so kraß, kamen dann auch 1918/19 vor und sind uns absolut unerklärlich. Im weitern unterschied sich die Grippe-Epidemie der 90er Jahre in zwei wesentlichen Punkten von derjenigen von 1918: In den 90er Jahren waren die Grippe-Opfer alles Patienten entweder des Säuglings- und Kleinkindesalters oder aber des hohen Alters, wobei das weibliche Geschlecht unter den Todesfällen eher etwas überwog. Überdies war die Influenza-Epidemie eine Krankheit der Wintermonate, während sie durch den Sommer hindurch fast völlig erlosch. Wenn ich immer wieder die Todesfälle an Influenza oder dem neueren Ausdruck Grippe erwähne, so deshalb, weil die Übergänge zwischen einem leichten Unwohlsein, einem kurzen Katarrh und einer schweren Bronchopneumonie so fließend sind, daß sämtliche Erkrankungen gar nicht erfaßt werden können, und daß, wie schon deutsche Ärzte 1918 festgestellt haben, allein die Zahl der 
Todesfälle Auskunft geben kann über die Verbreitung und Intensität der Epidemie. Weil die Epidemie der 90er Jahre nur im Winter aktiv war, wurden keine Militärpersonen davon erfaßt, da die damaligen militärischen Kurse alle nur in der guten Jahreszeit stattfanden.

\section{Die Grippe-Epidemie von 1918/19}

Diese unterschied sich von der noch den älteren Ärzten bekannten der 90er Jahre in erster Linie dadurch, daß es zuerst eine Sommerkrankheit war und daß sie ganz exquisit die Leute in den besten Jahren zwischen 20 und 40 weitaus am meisten betraf, d.h. genau das militärpflichtige Alter mit einem deutlich stärkeren Befall der Männer. Sie trat 1918 in Form einer Erkältungskrankheit mit etwas Fieber und Tracheitis bereits im Mai auf, im Militär z. B. bei einem Genfer Regiment, das dann gerade entlassen wurde, und dann bei Wachttruppen in Luzern, doch hatte man durchaus den Eindruck einer harmlosen Angelegenheit. Die Militärversicherung verzeichnete z.B. im Monat Juni 19183 Todesfälle an einer gewöhnlichen Pneumonie. Wenn wir berücksichtigen, wie leicht man sich im Militär erkältet und damals ohne Antibiotika und Chemotherapeutika die Pneumonie eine lebensgefährliche Krankheit darstellte, so fiel diese Zahl niemandem auf. Dann plötzlich, mit dem Beginn des Monats Juli 1918, veränderte sich schlagartig der Charakter der Krankheit: es traten schwere Fälle mit Komplikationen auf, die zum Tode führten, sowohl im Militär wie bei der Zivilbevölkerung. Bei der Armee ist natürlich zu berücksichtigen, wie groß der Bestand der aufgebotenen Truppen war. Derselbe war nämlich gar nicht etwa beeindruckend: Von den 365000 Mann, die die gesamte Armee umfaßte, waren vom Juni bis Ende 1918 bloß 33000-37000, dann 20000 und zuletzt nur noch 16000 Mann aufgeboten. Wie in ganz Mitteleuropa kam die Epidemie in der Gesamtschweiz in 2 Wellen, nämlich einer solchen mit einem kleineren Gipfel im Juli und dann einem sehr viel höheren im Spätherbst, wie wir aus der Kurve ersehen. Für die Zivilbevölkerung wurde in erster Linie ein Versammlungsverbot erlassen, das sich sogar auf die öffentlichen Gottesdienste und die großen Leichenbegängnisse erstreckte. Militärdienst ist nun gerade das Gegenteil eines Versammlungsverbotes, und deshalb wurde die Armee auch sehr stark befallen.

Bevor ich mich den Erkrankungen bei den Grenztruppen zuwende, möchte ich noch die Verhältnisse in den Rekrutenschulen darlegen. Aus den Militärakten des Bundesarchivs ist zu entnehmen, daß 13 aufgeführte 


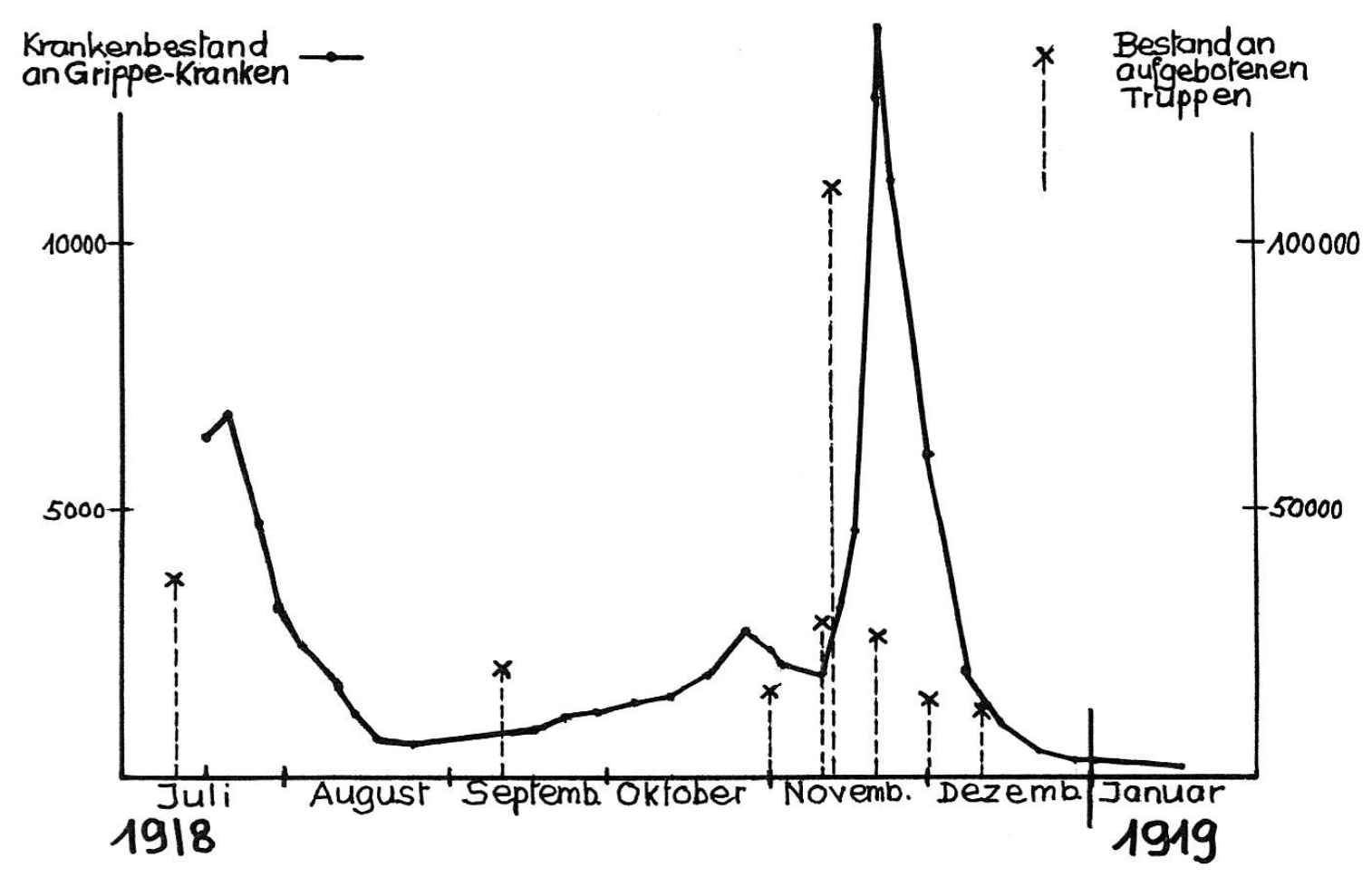

Abb. 1. Bestand an Grippekranken in der Armee und Zahl der aufgebotenen Truppen

Rekrutenschulen, die alle am 3. Juli eingerückt waren, in der Westschweiz mit ungeheurer Wucht von der Grippe erfaßt wurden. Von den Beständen, die alle zwischen 350 bis über 500 Mann betrugen, erlitten nämlich die Westschweizer Rekrutenschulen Lausanne, Colombier, Genf und Bern z.T. eine große Anzahl von Todesfällen. Am meisten war die Infanterie-Rekrutenschule Colombier betroffen, die eine Mortalität von 72,5\%o aufwies, d.h. von 483 eingerückten Rekruten verstarben 35 inkl. Schularzt. Die andern waren weniger stark befallen, wobei es auch hier Unterschiede gab, die unerklärlich sind. So starben in der Infanterie-Rekrutenschule Bern 4 Leute und in der viel kleineren Kavallerie-Rekrutenschule dagegen 8. In allen ostschweizerischen Rekrutenschulen, inkl. Thun und Aarau, waren nur ganz wenige oder meistens gar keine Todesfälle zu verzeichnen. Da die Rekruten natürlich aus dem Volke stammten, veranlaßte mich dieser Umstand, den Verhältnissen bei der Zivilbevölkerung nachzugehen, und ich bemühte mich, aller Akten darüber habhaft zu werden. Dabei zeigte es sich an den Beispielen des Kantons Neuenburg und der Stadt Bern, daß eben in der Westschweiz die schwere Epidemie im Juli stattfand und nicht im Spätherbst, und daß in der Ostschweiz, wo ich bloß eindeutige Zahlen für den 
Kanton Aargau bekam, die Verhältnisse gerade umgekehrt waren. Hier gab es im Juli fast keine Erkrankungen, und die großen Erkrankungs- und Todeszahlen traten erst im Spätherbst auf. Es gab somit einen westschweizerischen und einen ostschweizerischen Epidemietyp, wie wir aus der Karte ersehen können, wobei die Grenze merkwürdigerweise zwischen Bern und Thun verläuft. Die territoriale Verschiedenheit kommt am deutlichsten in den Zahlen für den Kanton Graubünden zum Ausdruck, indem völlig gleichartige Bezirke sich mit einer z.T. fünffachen Todesfallrate von benachbarten oder ähnlich gelegenen Bezirken unterschieden.

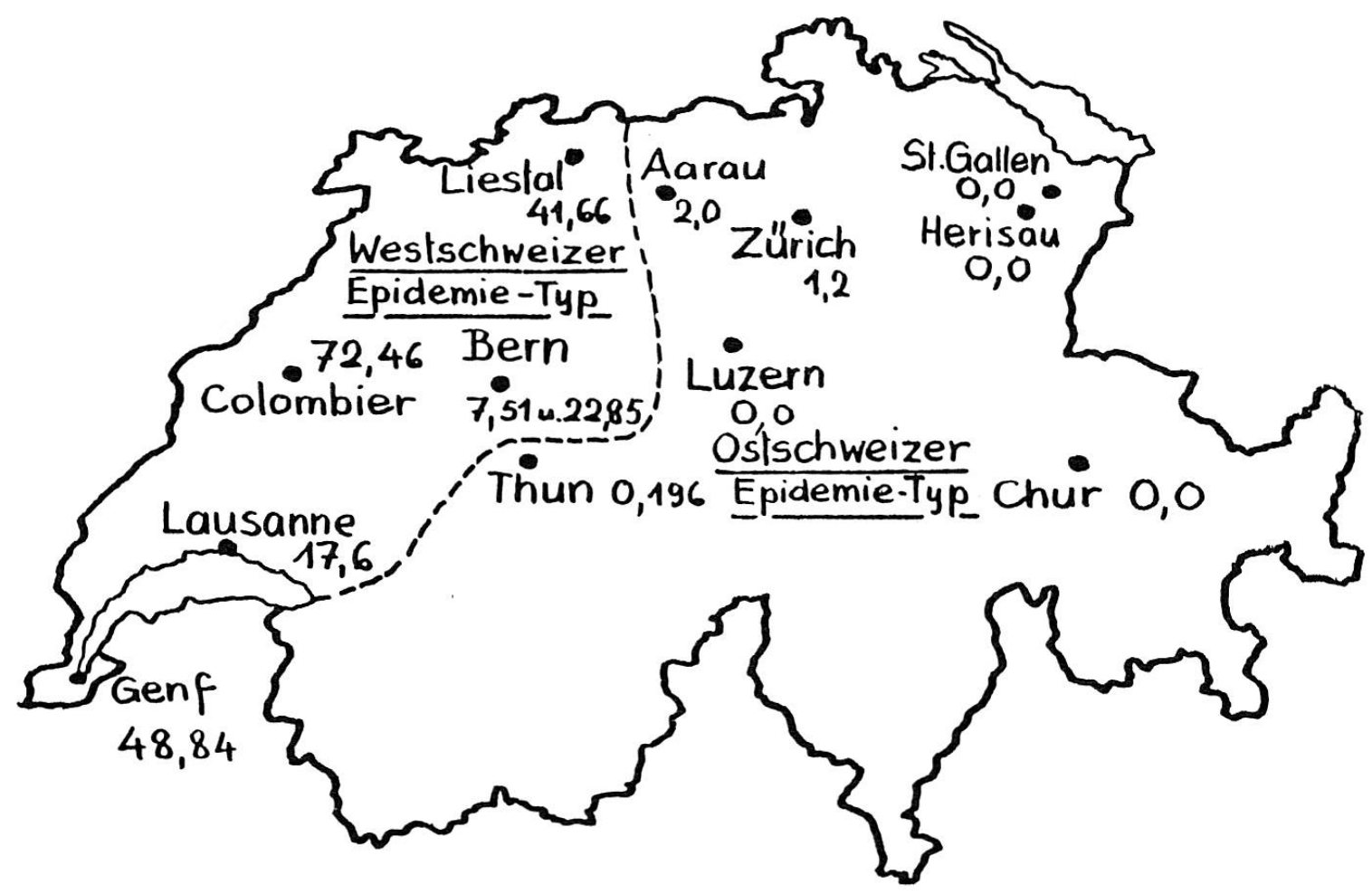

Abb.2. Mortalität der Juli-Rekrutenschulen 1918 in Promille des Gesamtbestandes

\section{Die Grippe-Epidemie bei den Grenztruppen}

Die Grippe ergriff diejenigen Grenztruppen, die im nördlichen Jura stationiert waren und von der 3. und 5. Division gestellt wurden, am meisten. Es erkrankten jeweils $40-80 \%$ der Soldaten, inkl. die zu ihrer Pflege aufgebotenen Sanitätseinheiten. Die Unterkunftsverhältnisse der Truppen waren im Jura fast durchwegs prekär. Von den Berner Truppen war die Gebirgsbri- 
gade 9 eingerückt und in der Ajoie verteilt, hatte zu wenig Ärzte und viel zu wenig Sanitätspersonal. Der Divisionsstab war völlig aufgelöst, und der Divisionsarzt, Oberst Rickli, sozialdemokratischer Nationalrat und Chefarzt des Bezirksspitals Langenthal, war nicht eingerückt, weil er im Spital der einzige Arzt war. Ein Bataillonsarzt mußte in Pruntrut die Stelle des Divisionsarztes versehen, dazu noch die Funktion eines Regimentsarztes ausüben und erst noch die Truppen seines eigenen Bataillons ärztlich versorgen! Katastrophal erwies sich, daß die zur Gebirgsbrigade 9 gehörende Gebirgs-Sanitäts-Abteilung nicht mit der Truppe eingesetzt war, sondern Dienst in den einzigen rückwärtigen Sanitätseinrichtungen der Armee, nämlich den Etappen-Sanitätsanstalten Solothurn und Olten, versahen. Der Armeearzt hatte sie dort eingesetzt, um das Aufgebot von Ambulanzen und Feldlazaretten, wie damals die Landwehr-Spitalabteilungen hießen, zu vermeiden. Erst nach einiger Zeit konnten dann im Juli in Pruntrut und Delsberg Krankendepots errichtet werden. Ebenso besorgniserregend war die Lage in Biel, indem dort am 8. Juli Jugendliche Unruhen verursachten, wobei gleich ein ganzes Gebirgs-Infanterie-Bataillon eingesetzt wurde und erkrankte und den Sanitätsdienst völlig überforderte. Die Komplikationen, die zum Tode führten, bestanden fast ausschließlich in Bronchopneumonien, die dann ein Herzversagen auslösten. Schon nach ein paar Tagen zeigte es sich, daß diejenigen Soldaten die Grippe am besten überstanden, die man ganz einfach auf dem Strohlager liegen ließ und nicht evakuierte bzw. keinem Transport aussetzte. Die Primitivität der Strohlager, die dann sehr viel Kritik in der Presse veranlaßte, erwies sich dabei keineswegs als nachteilig, indem im Vergleich dazu Truppen, wie z.B. Rekrutenschulen oder in Privathäusern untergebrachte Offiziere, in gleicher Weise oder noch stärker erkrankten. Man führte deshalb Transporte per Auto, die nur in kleiner Zahl vorhanden waren, nur auf kurze Strecken durch, und sämtliche Evakuationen in die Etappen-Sanitätsanstalten oder Zivilspitäler erfolgten dann mit Sanitätszügen. Ein weiterer Transport ins Innere des Landes, z. B. in leerstehende Hotels von Kurorten, erwies sich als völlig undurchführbar. Dazu fehlten auch Mittel. Es waren zwar 10 Militär-Sanitätsanstalten in der Truppenordnung vorgesehen, deren Kommandanten waren ernannt, jedoch hatten sie keine Truppen und keine Ausrüstung, weil ein diesbezügliches Gesuch des Armeearztes 1916/17 nach elfmonatigem Liegenbleiben praktisch abgelehnt worden war! Der Generalstabschef von Sprecher bedauerte dann in seinem Bericht über den Aktivdienst das Fehlen dieser Einrichtungen während der Grippe-Epidemie. Viel zu reden gab auch die Erkrankung 
des Gebirgs-Infanterie-Regimentes 6 der 1.Division, das vom Wallis aus über den Col des Mosses, Château-d'CEx und Freiburg in den Jura hätte marschieren sollen. Es wurde unterwegs in Aigle und in Château-d'Ex, wo eine Interniertenstation eingerichtet war, von der Grippe befallen, und der erste Todesfall unter den Internierten erfolgte 6 Stunden nach Eintreffen des Regimentes. Im Gegensatz zur 3. Division war jedoch der Divisionsarzt der 1.Division auf seinem Posten und organisierte sogleich in Aigle ein Krankendepot und ließ von Château-d'CEx aus die Leute ohne Gepäck nach Freiburg marschieren, wo der Marsch gestoppt wurde. Dort wurde in der Kaserne ein Krankendepot eingerichtet und dazu eine Sanitätskompagnie aufgeboten. Diese bezog um 6.30 Uhr die Kaserne, und um 8.30 Uhr kamen die ersten Kranken an, deren Zahl am gleichen Abend auf 275 anstieg. Es wurden auch $21 \mathrm{Krankenschwestern} \mathrm{angefordert,} \mathrm{die} \mathrm{am} \mathrm{5.} \mathrm{Juli} \mathrm{eintrafen.}$ Vom 7. bis 8. Juli stieg die Zahl der Kranken auf 900, so daß eine Filiale im Schulhaus Neuveville eingerichtet werden mußte und 2 Zivilärzte beigezogen wurden. Im ganzen wurden in diesen beiden Notspitälern etwa 1600 Kranke gepflegt, wovon nur 6 starben. Kein anderer von der Grippe befallener Truppenkörper wies so wenig Todesopfer auf. Vom 10. Juli an konnten schon 2 Rekonvaleszentenstationen in der Umgebung von Freiburg eingerichtet werden.

Die Schwierigkeiten in der gleichzeitigen ärztlichen Versorgung von erkrankter Zivilbevölkerung und Armee

Obschon nur rund $10 \%$ der gesamten Armee überhaupt eingerückt und der Grippe-Epidemie ausgesetzt waren, gab es in der ärztlichen Betreuung der Armee und der Zivilbevölkerung in vielen Kantonen große Schwierigkeiten. Viele Militärärzte erkrankten selbst und, wenn für sie Ersatz aufgeboten wurde, so reichten fast durchwegs die zivilen Behörden Dispensationsgesuche ein, da die neu aufgebotenen Ärzte wegen ihrer dringenden Aufgabe, die Zivilbevölkerung zu betreuen, nicht entbehrt werden konnten. Dabei stand wenigstens im größeren Teil der Schweiz die Höchstzahl der zivilen und militärisch Erkrankten, die erst im Spätherbst von der Krankheit erfaßt wurden, noch bevor. Es wäre nicht auszudenken, wenn bereits im Juli derartige Verhältnisse wie im Herbst geherrscht hätten!

An den 1918 gemachten Erfahrungen zeigt es sich, daß Armee und Zivilbevölkerung bei Kriegen und Epidemien nicht losgelöst voneinander sanitätsdienstlich betreut werden können. Die Unterstellung des gesamten 
Sanitätsdienstes unter den Rat für Gesamtverteidigung und die Schaffung eines gemeinsamen Koordinierten Sanitätsdienstes, wie er seit 1981 nun allmählich in die Tat umgesetzt wird, entspricht einer absoluten Notwendigkeit.

\section{Die politische Bedeutung der Grippe-Epidemie}

Die Mißstände im Armee-Sanitätsdienst erwiesen sich im Jahr des Klassenkampfes und des Generalstreikes für die militante Linkspresse als ein besonders gutes Objekt, um die bürgerliche Armeeführung anzugreifen. Merkwürdigerweise wurde der Armeearzt Oberst Hauser, der ein sehr tüchtiger Mann war, aber in der damaligen Sanitätsorganisation der Armee fast keine Kompetenzen hatte, zum Prügelknaben der Linken gemacht und nicht etwa der politische Exponent, der freisinnige Chef des Militärdepartementes, Bundesrat Décoppet. Die beiden Führer der extremen Linken in Basel, Dr. Welti und Fritz Schneider, der spätere Verwalter der Öffentlichen Krankenkasse Basel, verlangten sogar eine Militärstrafuntersuchung gegen Oberst Hauser, und auch die Geschäftsleitung der freisinnig-demokratischen Partei beantragte eine Untersuchung der Zustände. Überdies gelangte Oberst Hauser selbst an den General mit dem Ansuchen um eine Untersuchung gegen sich selbst, da er von der Linkspresse in maßloser Weise angegriffen wurde. Man sprach von «Hauserei», man sprach von «der Armeearzt trete ab!», und Welti und Schneider schrieben sogar, es wäre ein Verbrechen, diesen Mann auch nur noch eine Stunde im Dienst zu belassen. Die militärische Neutralitätskommission des Nationalrates gelangte an den Bundesrat mit dem Ersuchen, eine Untersuchungskommission einzusetzen. Durch eine Indiskretion, die an den «Bund» gelangte, enthielt das angebliche Communiqué der Neutralitätskommission eine gewisse Spitze gegen den General, der nämlich seinerseits schon Leute bestimmt hatte, um eine Untersuchungskommission zu bilden. Der General war dadurch dermaßen beleidigt, daß er am 31.Juli 1918 sein Rücktrittsgesuch einreichte, ohne auch nur die nächste Ausgabe des «Bund» abzuwarten, die dann den offiziellen Text enthielt, dem keinerlei Anfeindung der Armeeoberleitung zu entnehmen war. Nur mit Mühe konnte der frühere Korpskommandant und Präsident der militärischen Neutralitätskommission, Nationalrat Bühlmann von Großhöchstetten, den General umstimmen, sein Rücktrittsgesuch wieder zurückzuziehen.

Im übrigen war für die Sozialdemokratie in keiner Weise das Mitleid um die erkrankten und z.T. schlecht betreuten Soldaten das Motiv ihrer 
Aktionen. Als im Spätherbst wegen des Generalstreiks Ordnungstruppen aufgeboten werden mußten und viele Wehrmänner im Dienst an der Grippe erkrankten und viele daran starben, tönte es in den Kreisen der Linken ganz anders: Nun herrschte kein Bedauern mehr, vielmehr gab der kommunistische Arzt Brupbacher aus Zürich in seinem Tagebuch der Hoffnung Ausdruck, daß die Grippe die Soldaten dann kriegsmüde machen würde!

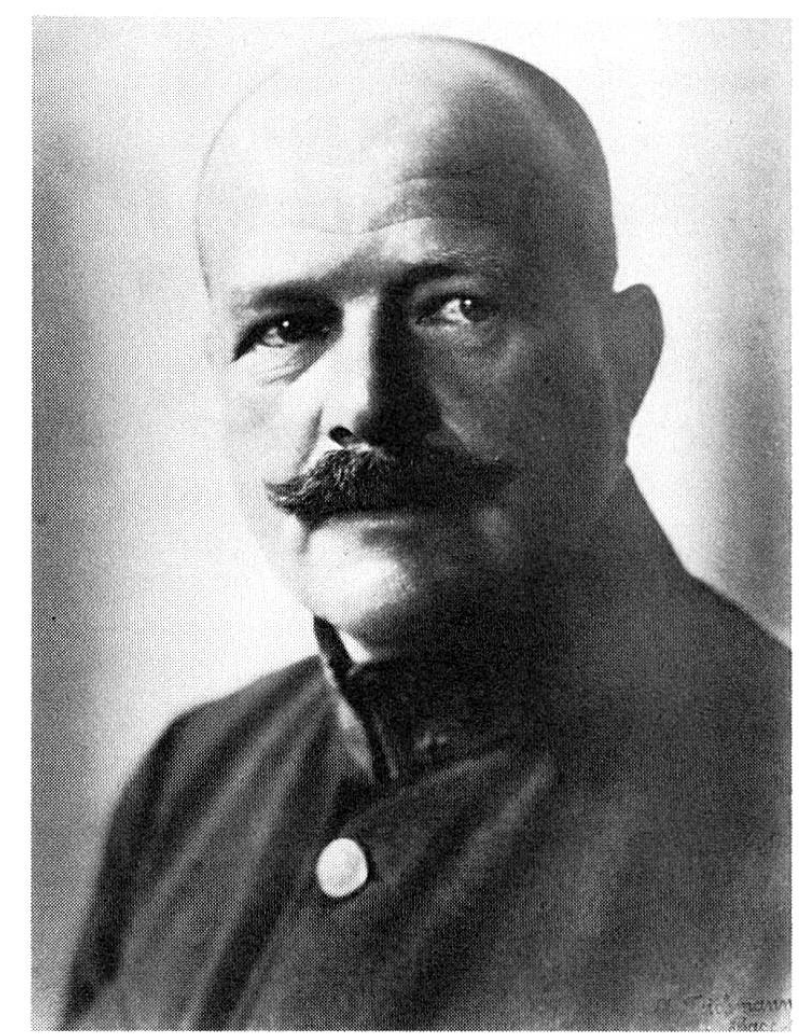

Abb.3. Oberst Dr. Carl Hauser, von Fontaines NE, 1866-1956

Oberfeldarzt von 1910 bis 1935

Reproduktion mit Bewilligung der Universitätsbibliothek

Basel und der Schweizerischen Landesbibliothek

Der Krankheitsverlauf der Grippe-Infektion in der Armee und die Ausdehnung der Epidemie unter den Wehrmännern

Über den klinischen Verlauf der Erkrankung beim Militär verfügen wir nur über zwei aussagekräftige Aktenstücke:

Einmal besitzt das Bundesamt für Sanität einen einzigen Bericht aus dieser Zeit, nämlich denjenigen von Oberlt. Krafft, der im Schulhaus Villeret 
im Sankt-Immer-Tal vom 1.Juli bis zum 2. August 1918 ein Feldspital führte, wo er 500 Kranke behandelte, von denen 30 verstarben. Dieser sehr tüchtige Sanitätsoffizier - 1890 geboren - wanderte nach dem Kriege nach Frankreich aus, wo er sich auch naturalisierte, den Arztberuf aufgab und sich der Literatur und der Dichtung widmete. 1944 promovierte er in Paris sogar noch in Literatur und verstarb 1960 in der Bretagne.

Ein anderer sehr schöner Bericht stammt vom Abteilungsarzt der Guidenabteilung 5 und wurde publiziert im «Correspondenzblatt für Schweizer Ärzte» im Herbst 1918 (Vorgänger der «Schweizerischen medizinischen Wochenschrift»). Der Verfasser war ein Leutnant Schinz, und der ausgezeichnet verfaßte Bericht veranlaßte mich, nach dem Verfasser zu fahnden, der sich als der spätere Pionier für Röntgendiagnostik, Prof. Schinz in Zürich, entpuppte. Er betreute 1918 die 2 Schwadronen der Guidenabteilung 5, die in Rümlang und Oberglatt einquartiert waren und die sich an zwei verschiedenen Quellen an der Grippe infiziert haben mußten. Jedenfalls war der Verlauf bei der einen Schwadron außerordentlich schwer, mit Todesfällen, während die andere recht glimpflich davonkam (siehe Graphik aus dem Bericht von Schinz).

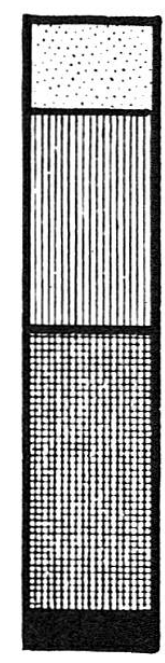

Schwadron in Oberglatt

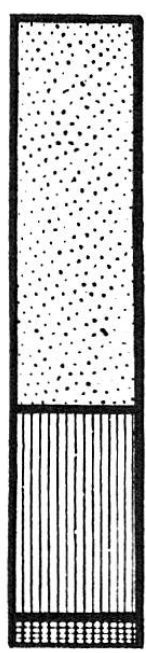

Schwadron in Rümlang

Abb.4. Erkrankung der Guidenabteilung 5 im Glatt-Tal (punktiert: Influenza abortiva, senkrecht schraffiert: Influenza mit Fieber, gerade schraffiert: Influenza Bronchitis und Pneumonie, schwarz: Todesfälle infolge Influenza-Pneumonie) 
Klinisch war der Verlauf, wie schon erwähnt, außerordentlich vielfältig: vom leichten Unwohlsein mit etwas Fieber, Husten und Halsschmerzen gab es alle Übergänge zur schwersten Bronchopneumonie mit Herzversagen als Folge der von den Begleit-Bakterien erzeugten Toxine. Die Inkubationszeit betrug wenige Stunden bis 2-3 Tage. Oberlt. Krafft beschrieb einen Fall, bei welchem vom Beginn der ersten Krankheitssymptome bis zum vasomotorisch bedingten Exitus nur eine halbe Stunde vergangen sei! Die Dauer der Krankheit war beim einzelnen Patienten ebenfalls ganz unterschiedlich und dauerte von wenigen Tagen bis zu mehreren Wochen, wobei keine Immunität entstand und die Rückfälle besonders gefährlich waren. Auch die negative Beeinflussung vorbestehender Krankheiten, insbesondere der Tuberkulose, wirkte sich oft besonders verhängnisvoll aus. Die Durchseuchung einer Einheit betrug nach Schinz etwa 20 Tage.

Die damalige Behandlung - vor der Ära der Antibiotika - bestand in erster Linie aus Bettruhe, Verabfolgung von Fiebermitteln und Expektorantien, Schröpfen und Wickeln.

Die Zahl der an Grippe krank gemeldeten Wehrmänner schwankte nach Einführung einer systematischen Zählung von Mitte Juli an bis Mitte November meist zwischen 5850 und 4500 täglich mit einem Minimum (Ende der ersten Infektionswelle) zwischen Mitte August und Mitte Oktober, wobei die niedrigste Zahl am 17. August mit 765 festgestellt werden konnte. Seither stieg die Zahl der grippekranken Wehrmänner wieder allmählich an, um am 21. November 1918, zur Zeit des Aufgebotes der Ordnungstruppen anläßlich des Generalstreiks, ein Maximum von 14023 zu erreichen. Deren Pflege war jedoch viel besser durchzuführen als während der Juli-Epidemie mit Truppeneinsatz im Jura, indem die Ordnungstruppen in den großen Städten und ihrer Umgebung eingesetzt wurden, wo die nötige Anzahl Notspitäler errichtet werden konnte und überhaupt genügend Ressourcen für die Pflege vorhanden waren.

Besonders wichtig ist die Feststellung der Zahl der im November an Grippe verstorbenen Wehrmänner, indem bis heute behauptet wird, allein der Truppeneinsatz anläßlich des Generalstreiks hätte 3000 Grippe-Todesopfer in der Armee gefordert. Dieser Auffassung muß entschieden entgegengetreten werden. Anhand des noch vorhandenen Totenrodels des Bundesamtes für Militärversicherung, den ich persönlich einsehen konnte, ergaben sich folgende Zahlen von Todesfällen in der Armee, die auf die Grippe zurückzuführen waren: 
1918: Juni: 1, Juli: 379, August: 62, September: 81, Oktober: 189, November: 807, Dezember: 248.

1919: Januar-Mai: 38.

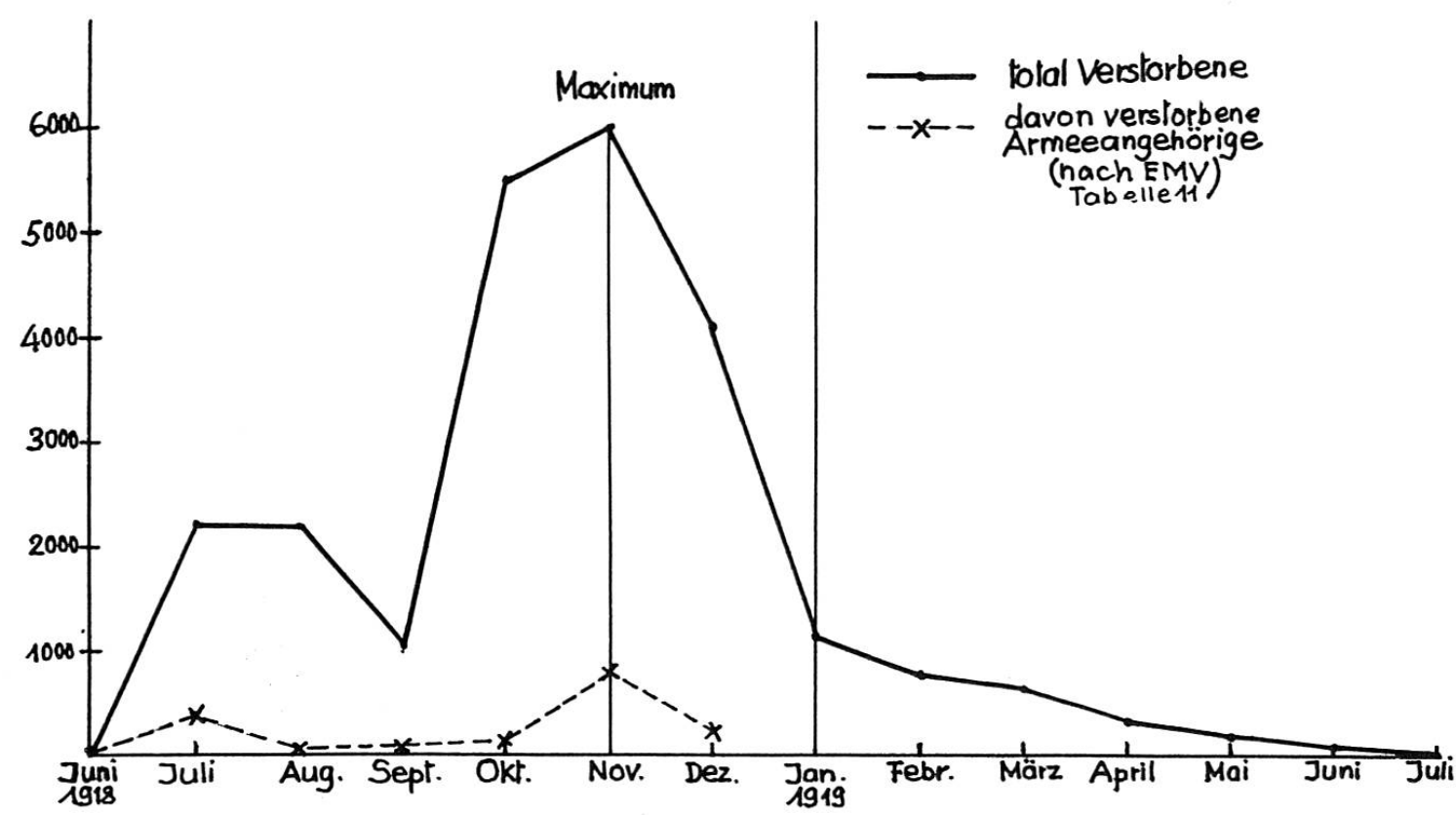

Abb.5. Sterbefälle an Grippe nach Monaten 1918 und 1919 für die ganze Schweiz mit Eintragung der verstorbenen Wehrmänner

Total verstarben also an der Grippe 1918/19 1805 Wehrmänner; unter den Ordnungstruppen während des Generalstreiks waren es 926 ! Somit erweist sich die Vorstellung von 3000 Generalstreik-Opfern eindeutig als Märchen, das durch keine Unterlagen gestützt werden kann. Dabei ist allerdings zu berücksichtigen, daß das Aufgebot der Ordnungstruppen zeitlich unglücklicherweise mit der hohen Erkrankungszahl unter der Zivilbevölkerung zusammenfiel. Sicher haben sich die Erkrankungen im Zivil und im Militär gegenseitig negativ beeinflußt, doch ist anzunehmen, daß viele aufgebotene Wehrmänner im November 1918 auch als Zivilisten gestorben wären.

\section{Das Wirken des Schweizerischen Roten Kreuzes}

Segensreich wirkte sich auch die Tätigkeit des Schweizerischen Roten Kreuzes unter der Leitung des Rot-Kreuz-Chefarztes, Oberst Bohny aus Basel, aus. Es wurden zur Pflege kranker Soldaten 742 Schwestern gestellt, 
wovon 63 in Erfüllung ihrer Pflicht verstorben sind. Auch viele freiwillige Samariterinnen wurden durch das Rote Kreuz eingesetzt, und gemeinsam mit der Militärversicherung konnten für die Genesung der grippekranken Soldaten wertvolle Rekonvaleszenten-Stationen im Berner Oberland, im Tessin und anderswo eingerichtet werden. Das Amerikanische Rote Kreuz war von der Grippe-Epidemie in der Schweiz so beeindruckt, daß es aus Dankbarkeit für das segensreiche internationale Wirken der Schweiz während des Krieges dem Schweizerischen Roten Kreuz die damals beachtliche Summe von 500000 Franken geschenkweise zur Verfügung stellte, welcher

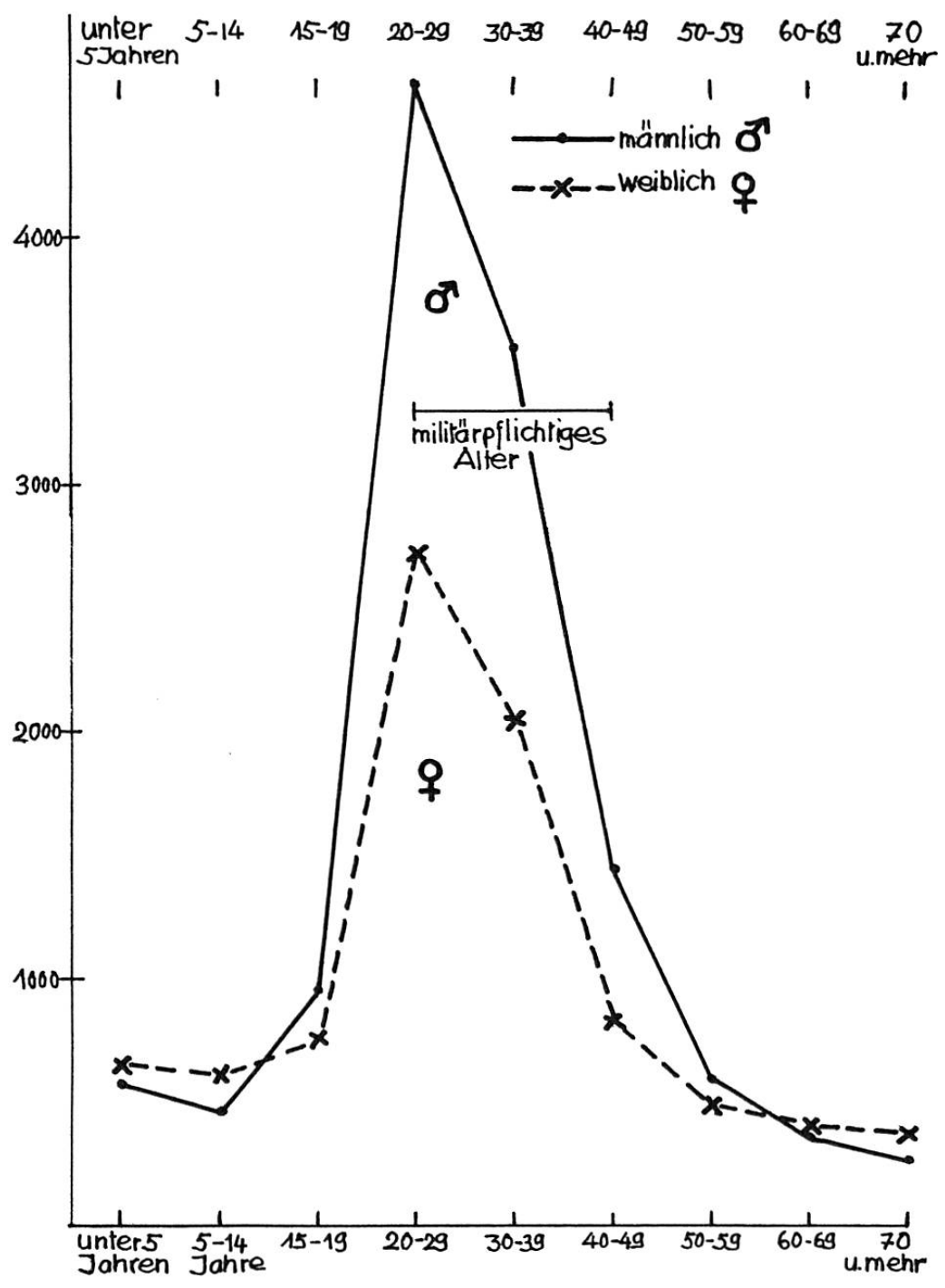

Abb. 6. Grippe-Todesfälle in der Schweiz 1918 nach Geschlecht und Alter 
Betrag wesentlich zum Ankauf der Liegenschaft Taubenstraße 8 in Bern, dem heutigen Sitz des Schweizerischen Roten Kreuzes, verwendet wurde.

Völlig ungeklärt bleibt wohl für immer die Frage nach der Zahl der wegen des Generalstreiks aufgebotenen Ordnungstruppen. Darüber herrscht noch heute ein fast unerschütterliches Tabu. Es fehlen die Angaben im Bericht des Generals über den Aktivdienst; im Bundesarchiv liegt einfach ein Blatt aus dem Jahre 1920 vor mit einer notierten Zahl von über 90000 Mann ohne jede Belege dazu und ohne Hinweis, wie die Zahl errechnet wurde. 1926 konnte anhand der Eintrittrapporte der Abteilung für Sanität eine Zahl von rund 50000 Diensttuenden nachgewiesen werden, doch sind die Akten dazu nicht mehr vorhanden, und andere Quellen wie Einrückungsbestände der kantonalen Landsturminfanterie bestehen nicht; die noch vorliegenden Tagebücher im Bundesarchiv sind unvollständig. Die große Zahl verstorbener Wehrmänner unter den Ordnungstruppen könnte theoretisch auf ein sehr großes Truppenaufgebot hinweisen, doch fiel - wie bereits erwähnt - das Aufgebot ausgerechnet mit dem hohen Herbstgipfel in der Zahl der verstorbenen Zivilisten im besten Mannesalter zusammen, was uns somit auch nicht weiterhilft. Diese unbekannte Zahl der aufgebotenen Ordnungstruppen im November 1918 wird aus politischen Gründen wohl immer ein Geheimnis der Schweizer Geschichte bleiben! 


\section{Zusammenfassung}

In der vorliegenden Darstellung wird der Verlauf der schweren Grippe-Epidemie in der Schweizerischen Armee der Jahre 1918/19 beschrieben. Die Armee war damals besonders stark befallen, indem die damalige Epidemie speziell Männer im Alter von 20 bis 40 Jahren erfaßte. Die Morbidität betrug bei gewissen Truppen bis $100 \%$ (Zivilbevölkerung insgesamt $56 \%$ mit einer Mortalität von 6,3\%o) und einer entsprechend hohen Mortalität. Der Armeesanitätsdienst war z.T. restlos überfordert, indem aufgebotene Sanitätseinheiten und Ärzte selbst erkrankten. Auch fehlte es teilweise an einer funktionstüchtigen Organisation, was heftige Angriffe der politischen Linken auf den damaligen Armeearzt Oberst Hauser auslöste. Eine erste Epidemiewelle im Sonner 1918 verursachte speziell viele Todesopfer in den Rekrutenschulen der Westschweiz. Die viel stärkere Erkrankungswelle im Spätherbst fiel mit dem großen Aufgebot an Ordnungstruppen anläßlich des Generalstreiks 1918 zusammen. Total verlor die Armee an Grippe-Toten 1805 Mann, wovon 926 auf die im November aufgebotenen Ordnungstruppen entfielen. Die Notwendigkeit des «Koordinierten Sanitätsdienstes», wie er 1981 eingeführt wurde, wird begründet. 


\section{Quellenverzeichnis}

Ungedruckte:

Originalakten des Bundesarchives in Bern: Grippe-Akten 1918/19 E/27 Akten Nr. 19714-19726, darunter speziell:

Wildbolz, Eduard, Oberstkorpskommandant: «Untersuchungen über die Grippe-Epidemie bei der 1. und 3. Division» vom 22. August 1918.

Krafft, Gustav, Oblt.: «Mémorial de l'hôpital de campagne de Villeret» August 1918. Im Bundesamt für Sanität EMD.

Eidgenössische Militärversicherung: Original-Totenrodel 1918/19.

Eine ganze Reihe persönlicher Mitteilungen aus verschiedenen Staats-Archiven diverser Kantone, Kantonsverwaltungen und statistischen und Gesundheitsämtern.

Gedruckte:

Staatsverwaltungsberichte des Kantons Bern. Staatsarchiv Bern.

Bolli, Beat, und Mitarbeiter: «Bericht der Kommission betreffend Untersuchung der Militärsanität» vom 24. Januar 1919, Bundesarchiv Bern.

Diverse Bulletins des Eidgenössischen Gesundheitsamtes, 1918/19.

«Correspondenzblatt für Schweizer Ärzte» 1918/19. Diverse Artikel über die Grippe und ihre Ätiologie und Behandlung, speziell von H. Sahli, Bern.

Frey, Friedrich E., «Die Influenza-Epidemie 1918 und 1919 im Kanton Aargau», Med.Diss. Zürich 1920.

Gautschi, Willi, «Der Landesstreik 1918», Einsiedeln/Zürich 1968.

Derselbe, «Geschichte des Kantons Aargau», III. Band, Baden 1978.

Kaufmann, Fritz, «Die Todesfälle der Schweizerischen Armee während der Mobilisation 1914-1918», Separatum aus Schweiz. med. Wochenschrift 1926, Nr.9.

Massini und Baur, in «Handbuch der Innern Medizin», 4. Auflage, Springer 1952.

Meuli, Oberfeldarzt: Ansprache anläßlich der Trauerfeierlichkeiten für Oberst Karl Hauser 1956, in «Vierteljahrsschrift Schweizerischer Sanitätsoffiziere» 1956, Heft 1, S. 56 ff.

Verwaltungsberichte des Bundesrates für 1918 und 1919, Abschnitt Militärversicherung.

Schweizerisches Rotes Kreuz, Jahresbericht 1918.

Schinz, Hans Rudolf, «Die Influenza-Epidemie bei der Guiden-Abteilung 5», Correspondenzblatt für Schweizer Ärzte 1918, S. $1329 \mathrm{ff}$. und 1374ff.

Schmid, Friedrich, «Die Influenza in der Schweiz 1889-1894, Bern 1894.

Statistisches Jahrbuch der Schweiz 1918 und 1938.

Berichte der Tagespresse 1918, speziell der «Berner Tagwacht» und der «Züricher Post».

Thalmann, Hans, «Die Grippe-Epidemie 1918/19 in Zürich», Med. Diss. Zürich 1968.

Wille, Ulrich, General, «Bericht an die Bundesversammlung über den Aktivdienst 1914-18», Zürich 1919.

Wyler, J., «Die Schweizerische Bevölkerung unter dem Einfluß des Weltkrieges», Zürich 1922.

Eine viel ausführlichere Arbeit über dasselbe Thema ist öffentlich im Manuskript zugänglich im Bundesarchiv Bern, in der Landesbibliothek Bern, im Staatsarchiv des Kantons Bern in 
Bern, im Bundesamt für Sanität EMD und in den Medizinhistorischen Instituten der Universitäten Bern und Zürich.

\section{Summary}

We describe the course of the serious epidemy of influenza in the Swiss army in 1918/19. The army was gravely struck, because the epidemy at that time infected mostly men of 20 to 40 years of age. In certain troups the morbidity increased to $100 \%$ with a corresponding high mortality. The civil population was infected in $56 \%$ with Mortality of $6,3 \%$. The sanitary service of the army was sometimes completely overcharged, because the sanitary troups and the physicians fell ill themselves. Moreover there was no sufficient organization, what caused vehement attacks of the political left against colonel Hauser, the chief physician of the army. The first outbreak of the epidemy in the summer 1918 caused a very great number of casualties among the recruits of the western part of Switzerland. A much stronger outbreak in late autumn of 1918 was simultaneous with the calling up of troups because of the general strike. In total the army lost 1805 men by influenza, of which 926 belonged to the troups called up in November. The necessity of the "coordinated sanitary service" introduced in 1981 is proved.

Dr. med. Walter Nussbaum

Feldeggweg 15

CH-3005 Bern 